\title{
A NOTE ON A THEOREM OF SPARR
}

\author{
Y. AMEUR
}

\begin{abstract}
We prove that, regardless of the choice of a positive, concave function $\psi$ on $\mathbf{R}_{+}$and a "weight function" $\lambda$, the weighted $\ell_{2}$-space $\ell_{2}(\psi(\lambda))$ is $c$-interpolation with respect to the couple $\left(\ell_{2}, \ell_{2}(\lambda)\right)$, where $c \leq \sqrt{2}$. Our main result is that $c=\sqrt{2}$ is best possible here; a fact which is implicit in the work of G. Sparr.
\end{abstract}

\section{A lemma on Pick functions}

Of general interest in the theory of interpolation spaces is the class $P^{\prime}$ of functions representable in the form

$$
h(\lambda)=\int_{[0, \infty]} \frac{(1+t) \lambda}{1+t \lambda} d \rho(t), \quad \lambda \in \mathbf{R}_{+},
$$

where $\rho$ is some positive Radon measure on $\mathbf{R}_{+}$. This class is known as the set of positive Pick functions on $\mathbf{R}_{+}$(cf. [2] or [4]). It is easy to see that $P^{\prime}$ constitutes a subcone of the convex cone of positive concave functions on $\mathbf{R}_{+}$.

In the following, it will be convenient besides (1) to work with a modified representation for $P^{\prime}$-functions (cf. [5], p. 266)

$$
h(\lambda)=\alpha+\beta \lambda+\int_{0}^{\infty} \frac{\lambda t}{\lambda+t} d \nu(t)
$$

where $\alpha \geq 0, \beta \geq 0$ and $v$ is a positive measure on $\mathrm{R}_{+}$such that $\int_{0}^{\infty} d v(t) /(1+$ $\left.t^{-1}\right)<\infty$.

We have the following basic lemma.

Lemma 1.1. Let $\psi$ be a positive concave function on $\mathbf{R}_{+}$. Then there exists a function $h \in P^{\prime}$ such that $h \leq \psi \leq 2 h$.

Proof (Cf. Peetre [11], bottom of p. 168.). It is well-known that an arbitrary positive, concave function can be represented in the form (cf. [3], p. 117)

$$
\psi(\lambda)=\alpha+\beta \lambda+\int_{0}^{\infty} \min (\lambda, t) d v(t)
$$

Received October 2, 2001; in revised form February, 2003. 
where $\alpha \geq 0, \beta \geq 0$ and $v$ a positive measure on $\mathbf{R}_{+}$such that $\int_{0}^{\infty} d v(t) /(1+$ $\left.t^{-1}\right)<\infty$. Next observe that for $\lambda, t>0$

$$
\frac{\lambda t}{\lambda+t} \leq \min (\lambda, t) \leq 2 \frac{\lambda t}{\lambda+t} .
$$

The lemma now follows from (2) and (3) on integration with respect to $v$.

\section{The Foiaş -Ong-Rosenthal question}

As we shall see presently, Lemma 1.1 is closely related to an interpolation theorem of Foiaş, Ong and Rosenthal [8], which goes back to the work of Jaak Peetre [10], [11]. Before we formulate this theorem, let us remind of some notions from the theory of interpolation spaces. (For more details on this theory, we refer to [3]).

Relative to a Hilbert couple $\overline{\mathscr{H}}=\left(\mathscr{H}_{0}, \mathscr{H}_{1}\right)$, we have the $K_{2}$-functional

$$
K_{2}(t, f)=K_{2}(t, f ; \overline{\mathscr{H}})=\inf _{f=f_{0}+f_{1}}\left(\left\|f_{0}\right\|_{0}^{2}+t\left\|f_{1}\right\|_{1}^{2}\right)^{1 / 2} .
$$

Let $\mathscr{H}_{0} \cap \mathscr{H}_{1}$ be dense in $\mathscr{H}_{0}$ and in $\mathscr{H}_{1}$. The basic fact for $K_{2}$ is the following (see e.g. [1]). Denote by $A$ the unbounded, densely defined, positive, injective operator in $\mathscr{H}_{0}$ such that

$$
\|f\|_{1}^{2}=(A f, f)_{0}, \quad f \in \mathscr{H}_{0} \cap \mathscr{H}_{1},
$$

then

$$
K_{2}(t, f)^{2}=\left(\frac{t A}{1+t A} f, f\right)_{0}
$$

With respect to $\mathscr{H}_{0}$ and $\mathscr{H}_{1}$ it will be advantageous to make use of several notations for the operator norms.

$$
\begin{aligned}
\|T\|^{2} & =\|T\|_{\mathscr{L}\left(\mathscr{C}_{0}\right)}^{2}=\sup _{(f, f)_{0} \leq 1}\left(T^{*} T f, f\right)_{0} \\
\|T\|_{A}^{2} & =\|T\|_{\mathscr{L}\left(\mathscr{L _ { 1 }}\right)}^{2}=\sup _{(A f, f)_{0} \leq 1}\left(T^{*} A T f, f\right)_{0} .
\end{aligned}
$$

Let $\mathscr{L}(\overline{\mathscr{H}})$ be the set of linear operators on $\mathscr{H}_{0}+\mathscr{H}_{1}$ such that the restriction of $T$ to $\mathscr{H}_{i}$ belongs to $\mathscr{L}\left(\mathscr{H}_{i}\right), i=0,1$. A Banach space norm on $\mathscr{L}(\overline{\mathscr{H}})$ is defined by

$$
\|T\|_{\mathscr{L}(\overline{\mathscr{C}})}=\max \left(\|T\|_{\mathscr{L}\left(\mathscr{H} \mathscr{C}_{0}\right)},\|T\|_{\mathscr{L}\left(\mathscr{H} \mathcal{C}_{1}\right)}\right)=\max \left(\|T\|,\|T\|_{A}\right) .
$$


We note that $K_{2}(t, \cdot)$ is an exact interpolation norm with respect to $\overline{\mathscr{H}}$, i.e.

(6) $K_{2}(t, T f) \leq\|T\|_{\mathscr{L}(\overline{\mathscr{H}})} K_{2}(t, f), \quad T \in \mathscr{L}(\overline{\mathscr{H}}), f \in \mathscr{H}_{0}+\mathscr{H}_{1}, \quad t>0$

which property is immediate from the definition of $K_{2}$. Given a positive, concave function $\psi$ on $\mathbf{R}_{+}$, let an intermediate Hilbert space $\mathscr{H}_{*}$ be defined as the completion of $\mathscr{H}_{0} \cap \mathscr{H}_{1}$ under the norm

$$
\|f\|_{*}^{2}=(\psi(A) f, f)_{0} .
$$

In accordance with (5) we shall use different notations for the operator norms

$$
\|T\|_{\psi(A)}^{2}=\|T\|_{\mathscr{L}\left(\mathscr{H}_{*}\right)}^{2}=\sup _{(\psi(A) f, f)_{0} \leq 1}\left(T^{*} \psi(A) T f, f\right)_{0} .
$$

By a theorem of Peetre [11], it is known that every positive, concave function $\psi$ on $\mathbf{R}_{+}$is an interpolation function of power 2 meaning that (for any $A, T$ )

$$
\max \left(\|T\|,\|T\|_{A}\right)<\infty \quad \text { implies } \quad\|T\|_{\psi(A)}<\infty .
$$

From the proof of Peetre's theorem, it can also bee deduced that there exists a constant $c \geq 1$ such that $\mathscr{H}_{*}$ is a $c$-interpolation space with respect to $\overline{\mathscr{H}}$ in the sense that

$$
\|T\|_{\psi(A)} \leq c \max \left(\|T\|,\|T\|_{A}\right), \quad T \in \mathscr{L}(\overline{\mathscr{H}}) .
$$

In 1972, Foiaş [6] noted that $c \leq 2$ for the best $c$. In a later paper, Foiaş, Ong and Rosenthal proved that $c \leq \sqrt{2}$, and also posed the question whether the constant $\sqrt{2}$ is best possible (cf. [8], question (i), p. 811). It is shown below that this is the case.

THEOREM 2.1. The best $c$ in (9) is $c=\sqrt{2}$.

Remark 2.2. This theorem is implicit in the work of Gunnar Sparr, cf. [12], Lemma 5.1. We shall here give a partially new proof, based on Lemma 1.1 and the following lemma.

Lemma 2.3. Every function $h$ in the class $P^{\prime}$ is exact interpolation in the sense that

$$
\|T\|_{h(A)} \leq \max \left(\|T\|,\|T\|_{A}\right), \quad T \in \mathscr{L}(\overline{\mathscr{H}}) .
$$

Remark 2.4. The above lemma is the easy half of a theorem of Foias and Lions [7] (see also [9]) which states that, for a positive function defined on $\mathbf{R}_{+}$, the condition $h \in P^{\prime}$ is equivalent to that $h$ fulfill (10) for every Hilbert couple $\overline{\mathscr{H}}$. 
Proof of Lemma 2.3.. Denote by $E$ the spectral measure of $A$ and let $\rho$ be the measure associated with $h$ as in (2). Then by (4)

$$
\begin{aligned}
\|f\|_{*}^{2} & =(h(A) f, f)_{0}=\int_{0}^{\infty}\left(\int_{[0, \infty]} \frac{(1+t) \lambda}{1+t \lambda} d \rho(t)\right) d\left(E_{\lambda} f, f\right)_{0} \\
& =\int_{[0, \infty]}\left(1+t^{-1}\right)\left(\int_{0}^{\infty} \frac{t \lambda}{1+t \lambda} d\left(E_{\lambda} f, f\right)_{0}\right) d \rho(t) \\
& =\int_{[0, \infty]}\left(1+t^{-1}\right) K_{2}(t, f ; \overline{\mathscr{H}})^{2} d \rho(t), \quad f \in \mathscr{H}_{0} \cap \mathscr{H}_{1} .
\end{aligned}
$$

It is easy to see that the latter expression extends to an exact interpolation norm with respect to $\overline{\mathscr{H}}$, viz. (10) holds (use (6) and integrate with respect to $d \rho(t)$ ).

Proof of Theorem 2.1. Referring to the smallest constant in (9), we first show that $c \leq \sqrt{2}$. Given an arbitrary concave, positive function $\psi$ on $\mathbf{R}_{+}$, let $h \in P^{\prime}$ be such that $h \leq \psi \leq 2 h$; then by Lemma 2.3,

$$
\begin{aligned}
\|T\|_{\psi(A)}^{2} & =\sup _{(\psi(A) f, f)_{0} \leq 1}\left(T^{*} \psi(A) T f, f\right)_{0} \leq \sup _{(h(A) f, f)_{0} \leq 1} 2\left(T^{*} h(A) T f, f\right)_{0} \\
& =2\|T\|_{h(A)}^{2} \leq 2 \max \left(\|T\|^{2},\|T\|_{A}^{2}\right), \quad T \in \mathscr{L}(\overline{\mathscr{H}}),
\end{aligned}
$$

and the estimate $c \leq \sqrt{2}$ follows incidentally. Proving $c \geq \sqrt{2}$ is more subtle; we shall require a clever three-dimensional argument due to G. Sparr, cf. [12], Example 5.3. Let $\mathscr{H}_{0}=\ell_{2}^{3}$ be the three-dimensional $\ell_{2}$-space. For $n \in \mathrm{N}$ let us put

$$
A_{n}=\left(\begin{array}{ccc}
\frac{1}{4 n^{2}} & 0 & 0 \\
0 & 1 & 0 \\
0 & 0 & 4 n^{2}
\end{array}\right), \quad g=\left(\begin{array}{l}
0 \\
1 \\
0
\end{array}\right), \quad f^{n}=\left(\begin{array}{c}
n \\
0 \\
\frac{1}{2}
\end{array}\right), \quad T_{n}=\left(\begin{array}{ccc}
0 & 0 & 0 \\
\frac{1}{2 n} & 0 & 1 \\
0 & 0 & 0
\end{array}\right)
$$

then $T_{n} f^{n}=g$ and a direct calculation yields that

$$
\left\|T_{n}\right\|=\left\|T_{n}\right\|_{A_{n}}=\sqrt{1+1 / 4 n^{2}}, \quad n \in \mathrm{N} .
$$

On the other hand, letting $\psi(\lambda)=\min (1, \lambda)$, we have

$$
\left\|T_{n} f^{n}\right\|_{\psi\left(A_{n}\right)}^{2}=\|g\|_{\psi\left(A_{n}\right)}^{2}=\left(\psi\left(A_{n}\right) g, g\right)_{0}=1, \quad n \in \mathbf{N},
$$

whereas

$$
\begin{aligned}
\left\|f^{n}\right\|_{\psi\left(A_{n}\right)}^{2}= & \left(\psi\left(A_{n}\right) f^{n}, f^{n}\right)_{0} \\
& =n^{2} \min \left(1,1 /\left(4 n^{2}\right)\right)+(1 / 4) \min \left(1,4 n^{2}\right)=1 / 2, \quad n \in \mathbf{N},
\end{aligned}
$$


and it follows that

$$
c \geq \frac{\|T\|_{\psi\left(A_{n}\right)}}{\sqrt{1+1 /\left(4 n^{2}\right)}} \geq \sqrt{\frac{2}{1+1 /\left(4 n^{2}\right)}} \nearrow \sqrt{2}, \quad n \rightarrow \infty .
$$

REMARK 2.5 (On Sparr's result). Let us introduce the modified $K_{2}$-functional

$$
L_{2}(t, f)^{2}=(\min (t, A) f, f)_{0} .
$$

By Sparr's work ([12], Lemma 5.1) it is known that

$$
K_{2}(t, g) \leq K_{2}(t, f) \quad \text { implies } \quad L_{2}(t, g) \leq L_{2}(t, \sqrt{2} f),
$$

where the constant $\sqrt{2}$ cannot be improved. Observe that, for an operator $T$, the condition $K_{2}(t, T f) \leq K_{2}(t, f), t>0$ is equivalent to that $\|T\|_{\mathscr{L}(\overline{\mathscr{H}})} \leq 1$. Moreover, by the representation (3) for a positive, concave function $\psi$, it is clear that

$$
\|f\|_{*}^{2}=(\psi(A) f, f)_{0}=\alpha\|f\|_{0}^{2}+\beta\|f\|_{1}^{2}+\int_{0}^{\infty} L_{2}(t, f)^{2} d v(t)
$$

with suitable $\alpha, \beta$ and $v$. Hence the condition $L_{2}(t, T f)^{2} \leq 2 L_{2}(t, f)^{2}, t>0$ implies that $\|T\|_{\psi(A)} \leq \sqrt{2}$. Thus (13) yields that (for all $A, T$ )

$$
\|T\|_{\mathscr{L}(\overline{\mathscr{H}})} \leq 1 \quad \text { implies } \quad\|T\|_{\psi(A)} \leq \sqrt{2},
$$

where the constant $\sqrt{2}$ is best possible. Note that this yields an alternative proof of Theorem 2.1.

We note the following, sharp version of Lemma 1.1.

THEOREM 2.6. The constant $c=2$ is smallest possible with respect to the property thet for any positive concave function $\psi$ on $\mathbf{R}_{+}$, there exists $h \in P^{\prime}$ such that $h \leq \psi \leq c h$.

Proof. Referring to the least constant, we have $c \leq 2$ by Lemma 1.1, and as in (12), one shows that for any positive concave $\psi$, any $A, T$

$$
\|T\|_{\psi(A)}^{2} \leq c \max \left(\|T\|^{2},\|T\|_{A}^{2}\right) .
$$

By Theorem 2.1, the smallest possible $c$ in the latter inequality is $c=2$.

\section{A note on $K_{2}$-functors}

We consider an application of Lemma 1.1 to the more functorial aspects of the theory. 
Given a positive Radon measure $\rho$ on $[0, \infty]$, let an interpolation functor $K_{2}(\rho)$ be defined on the category of Banach couples by

$$
\|f\|_{K_{2}(\rho)(\overline{\mathscr{A}})}=\left(\int_{[0, \infty]}\left(1+t^{-1}\right) K_{2}(t, f ; \overline{\mathscr{A}})^{2} d \rho(t)\right)^{1 / 2} .
$$

(Here the function $k: t \mapsto\left(1+t^{-1}\right) K_{2}(t, f)^{2}$ is defined by continuity at the points 0 and $\infty, k(0)=\|f\|_{1}^{2}$ and $k(\infty)=\|f\|_{0}^{2}$ where we have used the convention: $\|f\|_{i}=\infty$ if $f \notin \mathscr{H}_{i}, i=0,1$.)

COROLlary 3.1. Let $\overline{\mathscr{H}}$ be a regular Hilbert couple with associated operator A. Then, given any positive, concave funtion $\psi$, there exists a positive Radon measure $\rho$ on $[0, \infty]$ such that

$$
(1 / \sqrt{2})\|f\|_{K_{2}(\rho)(\overline{\mathscr{H}})} \leq\|f\|_{\psi(A)} \leq \sqrt{2}\|f\|_{K_{2}(\rho)(\overline{\mathscr{H}})}, \quad f \in K_{2}(\rho)(\overline{\mathscr{H}}),
$$

where the constant $\sqrt{2}$ cannot be improved.

Proof. This follows easily from Theorem 2.6 and (11).

\section{REFERENCES}

1. Ameur, Y., The Calderón problem for Hilbert couples, Ark. Mat. 41 (2003), 203-231.

2. Aronszjajn, N., and Donoghue, W., On exponential representations of functions, J. Analyse Math. 5 (1956-57), 321-388.

3. Bergh, J., and Löfström, J., Interpolation Spaces, an Introduction, Springer 1976.

4. Donoghue, W., Monotone Matrix Functions and Analytic Continuation, Springer 1974.

5. Donoghue, W., The interpolation of quadratic norms, Acta Math. 118 (1967), 251-270.

6. Foiaş, C., Invariant para-closed subspaces, Indiana Univ. Math. J. 21 (1972), 887-906.

7. Foiaş, C., and Lions, J.-L., Sur certains théorèmes d'interpolation, Acta Sci. Math. (Szeged) 22 (1961), 269-282.

8. Foiaş, C., Ong, S. C., and Rosenthal, P., An interpolation theorem and operator ranges, Integral Equations Operator Theory 10 (1987), 802-811.

9. Hughes, E., A short proof of an interpolation theorem, Canad. Math. Bull. 17 (1974), 127-128.

10. Peetre, J., On an interpolation theorem of Foiass and Lions, Acta Sci. Math. (Szeged) 25 (1964), 255-261.

11. Peetre, J., On interpolation functions, Acta Sci. Math. (Szeged) 27 (1966), 161-171.

12. Sparr, G., Interpolation of weighted $L_{p}$-spaces, Studia Math. 62 (1978), 229-271.

MATEMATISKA INSTITUTIONEN

UPPSALA UNIVERSITET

BOX 480

SE-751 06 UPPSALA

SWEDEN

E-mail: yacin@math.uu.se 